\title{
On the Rocking Behavior of Rigid Objects
}

\author{
FRANCISCO PRIETO* and PAULO B. LOURENÇO \\ Departamento de Engenharia Civil, Universidade do Minho, Guimaraes, Portugal
}

(Received: 23 October 2004; accepted in revised form: 8 June 2005)

\begin{abstract}
A novel formulation for the rocking motion of a rigid block on a rigid foundation is presented in this work. The traditional piecewise equations are replaced by a single ordinary differential equation. In addition, damping effects are no longer introduced by means of a coefficient of restitution but understood as the presence of impulsive forces. The agreement with the classical formalism is very good for both free rocking regime and harmonic forcing excitation.
\end{abstract}

Key words: Rocking Block, Dirac-delta, Complex.

Abbrevations: RM - Rocking Motion

\section{Introduction}

Rocking Motion (RM) is an oscillation characterized by an instantaneous shift between centres of rotation when a determined position is reached. At that point, an impulsive force takes place producing energy loss. The generic example is the case of a rigid prismatic block resting on a rigid foundation subjected to external earthquake loading. If friction is high enough to prevent sliding and the earthquake acceleration exceeds certain limit, the block will undergo rocking motion over its edges.

Although RM has been a subject of interest for researchers over a century, it was Housner [1] who presented the problem in a modern fashion. In that pioneer work it was showed that, despite of the apparent simplicity of a single block dynamics, a non-trivial behaviour was present and a number of unexpected results emerged.

Subsequent authors analysed the behaviour of a block subjected to the action of different earthquake inputs [2-5], while others focused their works on the stability of RM dynamics [6-8]. With respect to the latter subject, it has been shown how RM can become chaotic for some values of the parameters characterizing the system.

All the cited works make use of the formulation introduced by Housner [1] in terms of two differential equations, being each independent equation for each sign of the rotation angle. In addition, damping mechanisms are reproduced by a coefficient of restitution.

Nevertheless, Housner's piecewise formulation presents two important drawbacks. First, the theory makes the application of the standard mathematical theorems and

*Author for correspondence: fprieto@civil.uminho.pt 


\section{Francisco Prieto and Paulo B. Lourenço}

techniques of the theory of differential equations very difficult. Second, its generalization for a higher number of blocks becomes intractable when the number of degrees-of-freedom increases $[9,10]$.

The aim of the present contribution is to unify both piecewise equations and impact mechanisms into a model in which the motion is governed by a single differential equation.

The organization of the paper is as follows: In Section 2, Housner theory is revisited for comparison and reference. Section 3 introduces the complex formulation for both free and forced regimes. Finally, in Section 4, the results of the new formulation are tested with Housner theory.

\section{Housner Theory for Planar Rocking Motion}

To make the paper self-contained, a brief review for Housner [1] RM is included.

The equations of motion for a rigid rectangular block placed on a rigid base experiencing a time-dependent acceleration $a(\tau)$ (see Figure 1) are obtained from D'Alembert's principle [11]

$$
I \theta^{\prime \prime}-M R a(\tau) \cos (\alpha \mp \theta) \pm M g R \sin (\alpha \mp \theta)=0
$$

where $M$ is the mass, $I=(4 / 3) M R^{2}$, the corresponding moment of inertia (defined with respect to $\mathrm{O}$ or $\left.\mathrm{O}^{\prime}\right), g$ is the acceleration of gravity, and the geometrical parameters and coordinates are defined in Figure (1). The \pm sign refers to the domains $\theta>0$ and $\theta<0$ respectively.

Positive angles are defined counter-clockwise and time-differentiated variables are denoted by an apostrophe.

In eq.(1), $a(\tau)$ can represent any earthquake input but, for simplicity purposes, throughout this work harmonic forcing will be assumed, given by

$$
a(\tau)=\alpha g \beta \cos (\Omega \tau+\Phi)
$$

where $\beta$ and $\Omega$ represent the earthquake amplitude and circular frequency, respectively.

The following non-dimensional variables are now introduced

$$
x=\theta / \alpha, \quad t=p \tau, \quad \omega=\Omega / p,
$$

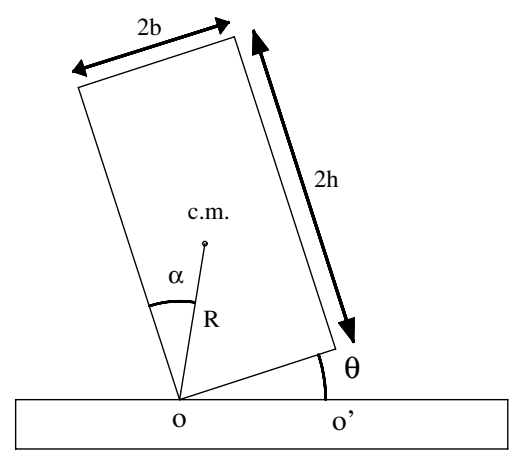

Figure 1. Geometry and notation for a rocking rectangular block. 
where $p=\sqrt{M g R / I}$. If differentiation with respect to the non-dimensional time $t$ is referenced by Newton's notation, equation (1) can be written in the following form

$$
|\ddot{x}|+\left(\frac{1}{\alpha}\right) \sin [\alpha(1-|x|)]-\operatorname{sign}(x) \beta \cos [\alpha(1-|x|)] \cos (\omega t+\Phi)=0
$$

Regarding the impact mechanisms, the piecewise theory assumes a coefficient of restitution that multiplies the angular velocity when the block passes through the equilibrium position at $x=0$. If $\dot{x}^{b}$ and $\dot{x}^{a}$ represent the angular velocities just before and after the impact respectively, Housner theory [1] states that,

$$
\dot{x}^{a} / \dot{x}^{b}=\mu \quad K^{a} / K^{b}=\mu^{2}
$$

where $\mu$ is the coefficient of restitution and $K$ is the kinetic energy of the block.

\section{Complex Formulation}

\subsection{Free Rocking Motion Without Impact}

In this section a Lagrangian function is built from the potential and kinetic energy expressions. Then, damping and harmonic excitation are included into the model through generalized forces in the D'Alembert's equations.

The potential energy of the block depends solely on the absolute value of the angle $\theta$ and reads

$$
U=M g R \cos (\alpha-|\theta|)
$$

In free rocking motion, under the actions of gravity and damping forces, a symmetry in the system exists, by which the dynamics are invariant under specular reflections with respect to a vertical line passing through the centre of rotation (see Figure 2).

The symmetry is equivalent to an invariance with respect to the sign of $\theta$, and, as a consequence, an associated conserved magnitude exists (Noether's Theorem [12]).

This fact can be exploited by expressing $\theta$ in complex form:

$$
\theta=r e^{i \psi}
$$

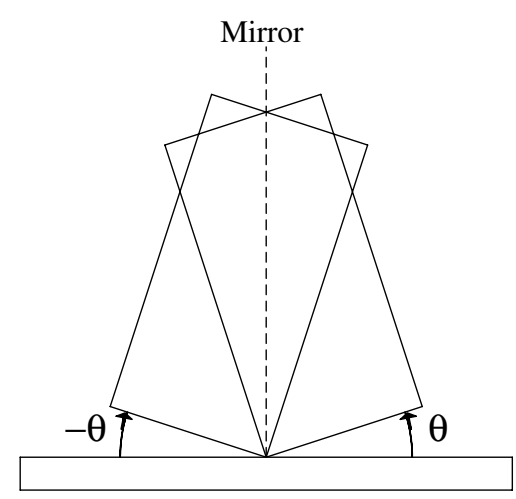

Figure 2. Dynamical symmetry associated to the sign of $\theta$. 


\section{Francisco Prieto and Paulo B. Lourenço}

Here, the sign of $\theta$ is only associated with $\psi$ and $r$ equals the absolute value of $\theta$. Therefore, in a heuristic manner, the following ( $\psi$-independent) kinetic energy can be proposed

$$
T=\frac{I}{2} \theta^{\prime} \theta^{*}
$$

where “*” means complex conjugate.

The Lagrangian function is built in the usual manner, as $L_{0}=T-U$ or

$$
L_{0}=\frac{I}{2}\left(r^{\prime 2}+r^{2} \psi^{\prime 2}\right)-M g R \cos (\alpha-r)
$$

This model can be set into direct analogy with either a two-body central problem in the complex plane or an inverted pendulum mechanism through simple variable transformations. However, the study of those mechanical analogies is outside the scope of the present paper.

The mechanical energy of the problem $E$ reads

$$
E=\frac{I}{2}\left(r^{\prime 2}+r^{2} \psi^{\prime 2}\right)+M g R \cos (\alpha-r)
$$

As mentioned above, due to the present symmetry, the Lagrangian function does not depend explicitly on $\psi$, and the canonical conjugate momentum $p_{\psi}$ turns out to be a well-defined constant quantity:

$$
p_{\psi}=\frac{\partial L_{0}}{\partial \psi^{\prime}}=I r^{2} \psi^{\prime}
$$

It is now assumed that $p_{\psi}$ will be referenced as $l_{0}: p_{\psi} \equiv l_{0}$

In its present form, the problem is equivalent to that of a single particle with kinetic energy $K=(I / 2) r^{\prime 2}$ moving in an effective potential $V_{\text {eff }}$ given by:

$$
V_{\text {eff }}(r)=\frac{l_{0}^{2}}{2 I r^{2}}+M g R \cos (\alpha-r)
$$

This potential adds an infinite repulsive barrier to $U(r)$, at $r=0$.

For a given value of energy $E$ the position of the corresponding turning points $r^{\min }$ and $r^{\max }$ can be obtained by numerically solving the equation $E=V_{\text {eff }}(r)$. However, for small $l_{0}$ the following (approximate) analytical solution can be derived for $r^{\min }$

$$
r^{\min }=\frac{l_{0}}{\sqrt{2 I\left(E-U_{0}\right)}}
$$

where $U_{0} \equiv U(r=0)=M g R \cos (\alpha)$.

\subsection{Implementations of Impact and Seismic Actions}

In much of what has been presented so far, $\mathrm{RM}$ has been considered under the assumption that both impact and seismic actions are neglected. However, until those interactions are included, no adequate understanding of the problem should be 
expected. Impact terms make the model no longer Hamiltonian, and then, generalized forces $Q_{q}$, accounting for such effects, should be considered. In the present case, this can be done via de D'Alembert equations:

$$
\begin{aligned}
& \frac{\mathrm{d}}{\mathrm{d} \tau}\left(\frac{\partial L_{0}}{\partial r^{\prime}}\right)-\frac{\partial L_{0}}{\partial r}=Q_{r}^{d}+Q_{r}^{e} \\
& \frac{\mathrm{d}}{\mathrm{d} \tau}\left(\frac{\partial L_{0}}{\partial \psi^{\prime}}\right)-\frac{\partial L_{0}}{\partial \psi}=Q_{\psi}^{d}+Q_{\psi}^{e}
\end{aligned}
$$

Here $Q_{r}^{d}$ and $Q_{r}^{e}$ represent the generalized damping and earthquake forces with respect to the canonical variable $r$ respectively. The same holds for $Q_{\psi}^{d}$ and $Q_{\psi}^{e}$ for $\psi$.

\subsubsection{Impact model. Phase dynamics}

Adopting again a heuristic approach, a reasonable form for $Q_{r}^{d}$ and $Q_{\psi}^{d}$ is proposed.

Taking into account the symmetry of the problem, it is assumed that

$$
Q_{\psi}^{d}=0
$$

Furthermore, the impulsive force is considered vertical and acting only through the edges $\mathrm{O}$ and $\mathrm{O}^{\prime}$ just at the moment of impact and with a magnitude equal to a half of the block weight $W=M g$ (see Figure 3). If the reaction force through an edge is referenced as $F$, it must be noticed that this force changes instantaneously in an amount of $W / 2$ during the impact. This suggests to consider the form of $F$ as:

$$
F=\frac{M g}{2} F_{\delta}
$$

being $F_{\delta}$ a Dirac-delta force.

The implementation of such an impulsive force has reported excellent results in a previous work [13].

On the other hand, since the generalized force with respect to $\theta$ (or to its absolute value $r$ ) is equal to the moment of the reaction force $F$ measured from the centres of rotation $\mathrm{O}$ and $\mathrm{O}$, the expression for $Q_{r}^{d}$ is simply: $Q_{r}^{d}=2 b F$, or, equivalently:

$$
Q_{r}^{d}=M g R \sin (\alpha) F_{\delta}
$$

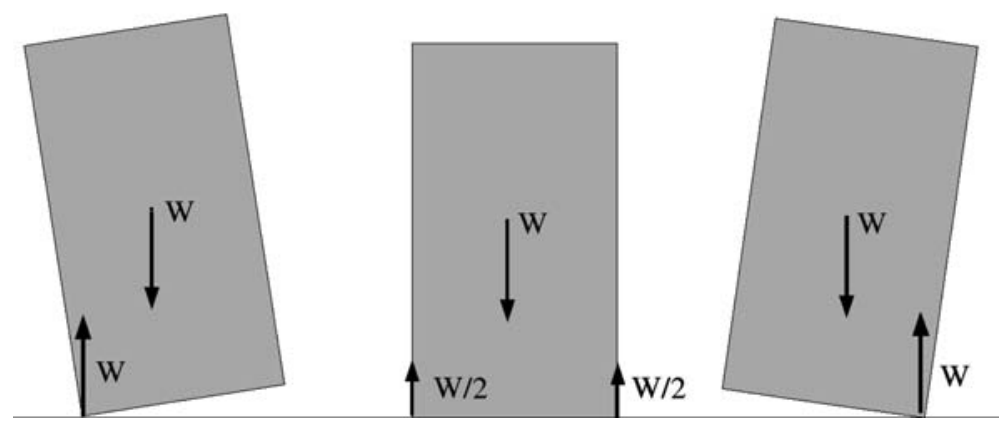

Figure 3. Impulsive forces during impacts. 


\section{Francisco Prieto and Paulo B. Lourenço}

In order to obtain the mathematical form of $F_{\delta}$, two issues should be taken into account. First, $\psi$ is associated to the sign of $\theta$ and, accordingly, $\psi^{\prime}$ is related to the change of the sign of $\theta$ with time. Second, the effect of the impact forces is to reduce the absolute value of the angular velocity when the change of sign takes place. is:

The simplest form for a generalized coupling between $r^{\prime}$ and the change of sign

$$
F_{\delta}=\sigma \psi^{\prime} r^{\prime}
$$

where $\sigma$ is a constant.

\subsubsection{Evaluation of the constant $\sigma$}

To obtain the value of $\sigma$ just introduced, the power dissipated by $Q_{r}^{d}$ is considered. Taking into account equations (3),(17) and (18) and recalling that the power $P$, dissipated by a damping force is equal to the product of that force by the velocity: $P \equiv$ $\mathrm{dE} / \mathrm{d} \tau=Q_{r}^{d} r^{\prime}$

$$
\frac{\mathrm{d} E}{\mathrm{~d} \tau}=r^{\prime} Q_{r}^{d}=I p^{2} \sigma\left(r^{\prime}\right)^{2} \sin (\alpha) \psi^{\prime}
$$

From equations(10-13) it holds that: $\left(r^{\prime}\right)^{2}=2\left(E-V_{\text {eff }}\right) / I$ and this can be substituted in equation (19) to give:

$$
\frac{\mathrm{d} E}{E-V_{\text {eff }}(r)}=2 p^{2} \sigma \sin (\alpha) \mathrm{d} \psi
$$

This expression must be integrated during an impact. The left-hand-side is integrated in energies between $E^{b}$ and $E^{a}$ (two consecutive impacts), while the right-hand-side is integrated in the phase $\psi$.

Since, at the impact, the contribution to the change of energy $E=K+V_{\text {eff }}$ due to $V_{\text {eff }}$ is negligible compared to that corresponding to the kinetic energy, $d V_{\text {eff }}$ can be neglected in the numerator of equation (20)

$$
\frac{\mathrm{d} E}{E-V_{\text {eff }}(r)}=\frac{\mathrm{d}\left(K+V_{\text {eff }}\right)}{K} \approx \frac{\mathrm{d} K}{K}
$$

leading after integration to

$$
\ln \left(\frac{K^{a}}{K^{b}}\right)=2 p^{2} \sigma \Delta \psi \sin (\alpha)
$$

Because, it also holds (see appendix, equation (56)) that for $l_{0} \rightarrow 0, \Delta \psi=\pi$, expressions (22) and (5) lead to

$$
\sigma=\frac{\ln (\mu)}{\pi p^{2} \sin (\alpha)}
$$

The form of the generalized damping force results in:

$$
Q_{r}^{d}=\left(\frac{\ln (\mu) I}{\pi}\right) \psi^{\prime} r^{\prime}
$$




\subsubsection{Implementation of harmonic forcing}

When a horizontal force is applied to the block, the symmetry introduced in Section 3.1 does not hold any more. The dynamics are no longer invariant under the change of sign of $\theta$, and, accordingly, $l_{0}$ becomes, in general, a function of time.

However, for $l_{0}$ sufficiently small, it will be shown that this variation is negligible.

The effect of the acceleration considered in equation (2) consists on a horizontal force of magnitude $-M a(\tau)$, applied at centre of mass of the block.

$$
\vec{F}=-M \vec{a}(\tau)
$$

As a consequence, the generalized forces corresponding to the variables $r$ and $\psi$ are

$$
\begin{aligned}
Q_{r}^{e} & =-M a(\tau) \frac{\partial X_{\mathrm{cm}}}{\partial r} \\
Q_{\psi}^{e} & =-M a(\tau) \frac{\partial X_{\mathrm{cm}}}{\partial \psi}
\end{aligned}
$$

being $X_{\mathrm{cm}}$ the horizontal coordinate of the centre of mass measured with respect to the centre of rotation:

$$
X_{\mathrm{cm}}=\operatorname{sign}(\theta) R \sin (\alpha-|\theta|)
$$

As shown in the appendix (see equation (58) and Figure (6)), for $l_{0}$ small, the sign of $\theta$ can be given by $\cos (\psi)$. Therefore, equation (28) renders

$$
X_{\mathrm{cm}}=R \cos (\psi) \sin (\alpha-r)
$$

which, once substituted in equations (26) and (27), leads to

$$
\begin{aligned}
& Q_{r}^{e}=M a(\tau) R \cos (\alpha-r) \cos (\psi) \\
& Q_{\psi}^{e}=M a(\tau) R \sin (\alpha-r) \sin (\psi)
\end{aligned}
$$

In the limit $l_{0} \rightarrow 0, \psi$ only takes the values $n \pi(n=0,1,2, \ldots)$ and $Q_{\psi}^{e}$ vanishes.

Now, the non-dimensional variable $\chi$, equal to $|x|$ and given by: $\chi \equiv(r / \alpha)$ is introduced. In addition, the Lagrangian function $L_{0}$ is normalized by the definition: $L \equiv$ $L_{0} /\left(I p^{2} \alpha^{2}\right)$. The new Lagrangian results in

$$
L=\frac{1}{2}\left(\dot{\chi}^{2}+\chi^{2} \dot{\psi}^{2}\right)-\frac{1}{\alpha^{2}} \cos (\alpha-\alpha \chi) .
$$

The corresponding normalized D'Alembert forces with respect to $\chi$, considering equations (2), (24) and (29) render

$$
\begin{aligned}
& Q_{\chi}^{d}=\left(I p^{2} \alpha\right)\left(\frac{\ln (\mu)}{\pi}\right) \dot{\psi} \dot{\chi} \\
& Q_{\chi}^{e}=\left(I p^{2} \alpha\right) \beta \cos (\omega t+\Phi) \cos (\alpha-\alpha \chi) \cos (\psi)
\end{aligned}
$$

Taking into account that;

$$
\begin{aligned}
& \frac{\partial L_{0}}{\partial r}=\left(I p^{2} \alpha\right) \frac{\partial L}{\partial \chi} \\
& \frac{\mathrm{d}}{\mathrm{d} \tau}\left(\frac{\partial L_{0}}{\partial r^{\prime}}\right)=\left(I p^{2} \alpha\right) \frac{\mathrm{d}}{\mathrm{d} t}\left(\frac{\partial L}{\partial \dot{\chi}}\right)
\end{aligned}
$$


D'Alembert equations hold

$$
\ddot{\chi}=\chi \dot{\psi}^{2}-\frac{1}{\alpha} \sin (\alpha-\alpha \chi)+\frac{\ln (\mu)}{\pi} \dot{\chi} \dot{\psi}+\beta \cos (\omega t+\Phi) \cos (\alpha-\alpha \chi) \cos (\psi)
$$

and

$$
\frac{\mathrm{d}}{\mathrm{d} t}\left(\frac{\partial L}{\partial \dot{\psi}}\right)=0
$$

This last equation simply expresses the fact that the quantity $l$, defined by $l \equiv \chi^{2} \dot{\psi}$ is a constant of the motion. In fact, $l$ is the non-dimensional analogous of $l_{0} ; l=$ $l_{0} /\left(I p \alpha^{2}\right)$.

An autonomous system of differential equations can be formed by embedding the space of variables in a higher dimension, defining:

$$
\left(\chi_{1}, \chi_{2}, \chi_{3}, \chi_{4}\right) \equiv(\chi, \dot{\chi}, \psi, \omega t)
$$

Due to the effect of a finite interaction, the integrator will take certain intermediate values for the velocity at the impact time. This average results in adding a factor of $1 / 2$ (for a more detailed discussion, please, see [13]).Therefore, a factor of 2 should be included in the impulsive term in order to make theoretical and numerical results compatible.

The final system of equations (where the earthquake phase $\Phi$ assumed to be zero) is

$$
\begin{aligned}
& \dot{\chi}_{1}=\chi_{2} \\
& \dot{\chi}_{2}=\frac{l^{2}}{\chi_{1}^{3}}-\frac{\sin \left(\alpha-\alpha \chi_{1}\right)}{\alpha}+\frac{2 \ln (\mu) \chi_{2} l}{\pi \chi_{1}^{2}}+\beta \cos \left(\chi_{4}\right) \cos \left(\chi_{3}\right) \cos \left(\alpha-\alpha \chi_{1}\right) \\
& \dot{\chi}_{3}=\frac{l}{\chi_{1}^{2}} \\
& \dot{\chi}_{4}=\omega
\end{aligned}
$$

\section{Comparison with Housner Theory}

For small $x$ and $\alpha$, analytical solutions do exist for equations (4) (see, for instance [7]). Therefore, it is possible to compare the novel Formulation of equations (37) with analytical expressions.

For numerical integration of system of equations (37), a 6-th order Runge-Kutta method has been used, taking into account that:

- The integration step must always be smaller than $l$. In the computer implementation, a value of $l / 10$ has been adopted as default.

- The value of $r$ (or its non-dimensional analogous $\chi$ ) is lower-bounded by the value $r_{c}\left(\right.$ or $\left.\chi_{c}\right)$ which corresponds to a minimum of the effective potential $V_{\text {eff }}$.

The value for $r_{c}$ or $\chi_{c}$ can be obtained by solving:

$$
\left.\frac{\mathrm{d} V_{\text {eff }}(r)}{d r}\right|_{r=r_{c}}=0
$$

which leads to the nonlinear equation 


$$
M g R \sin \left(\alpha-r_{c}\right)=\frac{l_{0}^{2}}{I r_{c}^{3}}
$$

In terms of $l$ and $\chi$, this equation leads to

$$
\sin (\alpha-\alpha \chi) \chi_{c}^{3}=\alpha l^{2}
$$

For small values of $\alpha$ and $\chi_{c}$, the following approximate value can be obtained

$$
\chi_{c} \approx l^{2 / 3}
$$

Therefore, a reasonable condition for numerical integration of the system of equations (37) is $\chi_{1} \geq l^{2 / 3}$.

Next, two different examples are used to validate the proposed novel formulations. The obtained results plot the real part of the non-dimensional angle $x$ versus the non-dimensional time $t$, that is $(t, \operatorname{Re}(x)=\chi \cos (\psi)))$, or equivalent, $\left(t, \chi_{1} \cos \left(\chi_{3}\right)\right)$.

As a first example, Housner theory for free rocking motion will be compared with the model for different values of $l$. Under these conditions, the performance of the damping term $Q_{\chi}^{d}$ is evaluated. Figure (4) illustrates the results, being clear that convergence to the theoretical solution is obtained upon increasingly lower values of $l$. The parameters used for Figure (4) are: $\alpha=\operatorname{tg}^{-1}(1 / 4), \mu=0.925$ and initial conditions; $\left(\chi_{1}(0)=0.5, \chi_{2}(0)=0.0, \chi_{3}(0)=0.0, \chi_{4}(0)=0.0\right)$.

The next example validates $Q_{\chi}^{d}$ and an "accurate" value of $l=10^{-5}$ was chosen for the calculation. Figure (5) illustrates the perfect agreement between the theoretical solution and the novel complex formulation. The parameters used now are: $\alpha=\operatorname{tg}^{-1}(1 / 4), \mu=0.925$, and initial conditions; $\left(\chi_{1}(0)=0.061, \chi_{2}(0)=-0.061\right.$, $\left.\chi_{3}(0)=0.0, \chi_{4}(0)=0.0\right)$.

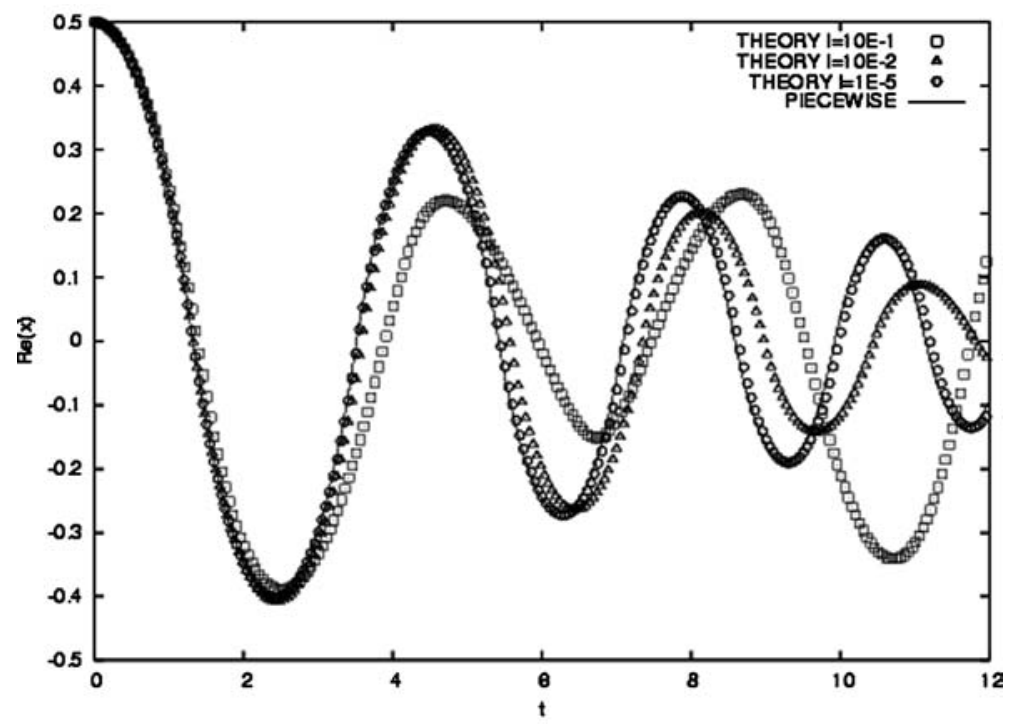

Figure 4. Comparison between Housner theory and complex formulation for different values of $l$ under free rocking motion. $\mu=0.925$ and $\alpha=\operatorname{tg}^{-1}(1 / 4)$ with initial conditions; $\left(\chi_{1}(0)=0.5, \chi_{2}(0)=0.0\right.$, $\left.\chi_{3}(0)=0.0, \chi_{4}(0)=0.0\right)$. 


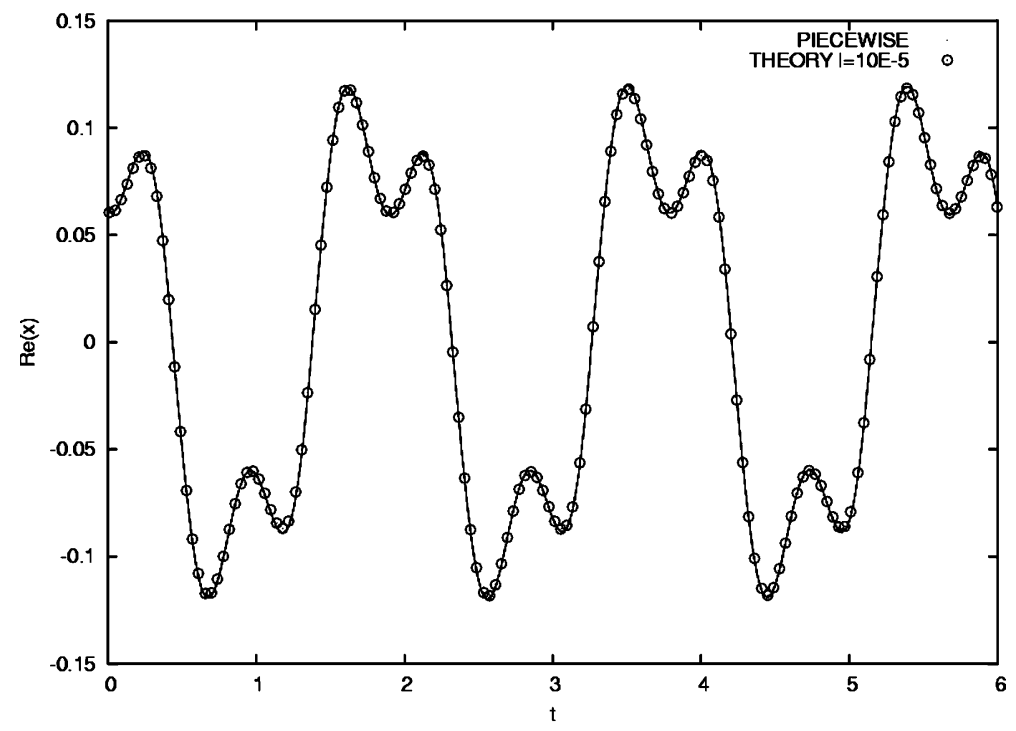

Figure 5. Comparison between Housner theory and complex formulation for $l=10^{-5}$ and parameters: $\alpha=\operatorname{tg}^{-1}(1 / 4), \mu=0.925$, and initial conditions; $\left(\chi_{1}(0)=0.061, \chi_{2}(0)=-0.061, \chi_{3}(0)=0.0, \chi_{4}(0)=0.0\right)$.

\section{Conclusions}

The complex formulation presented in this work unifies both piecewise equations of motion and impact mechanisms into a single model, in which the dynamics are governed by a unique differential equation. This approach allows further studies with a higher number of blocks and using statistical mechanics.

Damping effects are understood by means of a generalized force constructed in a heuristic manner. This force behaves as a Dirac-delta force, which is in agreement with the results reported elsewhere [13].

The model proposed can be set in direct analogy with either a two-body central problem in the complex plane or an inverted pendulum through simple variable transformations. The study of these mechanical analogies is outside the scope of the present paper.

\section{Appendix: Dirac-delta Forces and Phase Dynamics}

The aim of this appendix is to show that, during an impact, the quantity

$$
f_{l}(r) \equiv \frac{1}{\pi} \frac{\mathrm{d} \psi}{\mathrm{d} r}
$$

behaves as a Dirac-delta function.

The Dirac-delta function is defined in this work by its assigned properties:

$$
\int_{a}^{b} g(x) \delta\left(x-x_{0}\right)=\left\{\begin{array}{cc}
g\left(x_{0}\right) ; & x_{0} \in(a, b) \\
\frac{1}{2} g\left(x_{0}\right) ; & x_{0}=a, b \\
0 ; & \text { elsewhere }
\end{array}\right.
$$


As commonly assumed, $\delta(x)$ will be approximated by a limit sequence of continuous functions $\delta_{l}(x)$.

Strictly, it must be recalled that the limit

$$
\lim _{l \rightarrow 0} \delta_{l}(x)
$$

does not exist. Nevertheless, $\delta(x)$ may be treated consistently in the form (see, for instance, Arfken, [14])

$$
\int_{a}^{b} \delta(x) g(x) \mathrm{d} x=\lim _{l \rightarrow 0} \int_{a}^{b} \delta_{l}(x) g(x) d x
$$

Selecting $a=r^{\min }$, one obtains

$$
\lim _{l \rightarrow 0} \int_{r^{\min }}^{b} \delta_{l}\left(r-r^{\min }\right) g(r) \mathrm{d} r=\frac{1}{2} g\left(r^{\min }\right)
$$

From equations (10-12) and the definition of equation (42), the following expression is obtained:

$$
f_{l}(r)=\frac{l}{\pi r \sqrt{2(E-U) I r^{2}-l^{2}}}
$$

The key quantity to be computed is:

$$
\lim _{l \rightarrow 0} \int_{r^{\min }}^{b} f_{l}(r) g(r) \mathrm{d} r
$$

being $g(r)$ an arbitrary function and $r^{\min }$ given by equation (13).

Through a change of variable $y=r / r^{\mathrm{min}}$, and, after some manipulation, the following expression holds

$$
\lim _{l \rightarrow 0} \int_{1}^{\frac{b}{r \min }} \frac{g\left(y r^{\mathrm{min}}\right) \mathrm{d} y}{\pi y \sqrt{\left(\frac{E-U\left(y r^{\mathrm{min}}\right)}{E-U_{0}}\right) y^{2}-1}}
$$

Calculating the limit (note that $b$ is large, but finite), the result is

$$
\frac{g(0)}{\pi} \int_{1}^{\infty} \frac{\mathrm{d} y}{y \sqrt{y^{2}-1}}
$$

This integral can be calculated in the complex plane taking into account that the integrand has a simple pole at $y=0$ and two branch points at $y= \pm 1$, giving $\pi / 2$. Therefore

$$
\lim _{l \rightarrow 0} \int_{r^{\min }}^{b} f_{l}(r) g(r) \mathrm{d} r=\frac{g(0)}{2}
$$

Comparing the above expression with equation (45), since $r^{\min }$ and $l$ go simultaneously to zero, it is possible to obtain 


$$
\begin{aligned}
& \lim _{l \rightarrow 0} \int_{0}^{b} f_{l}(x) g(x) \mathrm{d} x=\frac{g(0)}{2} \\
& \lim _{l \rightarrow 0} \int_{0}^{b} \delta_{l}(x) g(x) \mathrm{d} x=\frac{g(0)}{2}
\end{aligned}
$$

From this behaviour of $f_{l}(x)$ on the arbitrary function $g(x)$, it results that $f_{l}(x)$ can be identified with a $\delta$-sequence;

$$
f_{l}(x) \approx \delta_{l}(x)
$$

and it is justified to assign:

$$
\frac{\mathrm{d} \psi}{\mathrm{d} \tau}=\pi \delta_{l}(\tau)
$$

From this equation, the dispersion of the $\psi$ angle at every impact of the block with the foundation can also be obtained, as

$$
\lim _{l \rightarrow 0} \Delta \psi=\pi \int_{-\infty}^{\infty} \delta(x) \mathrm{d} x=\pi
$$

By using the property of the Delta function (where the sum is extended over all the zeros $\left(t_{j}\right)$ of the function $x(t)$, e.g. the times of impact):

$$
\delta(x(t))=\frac{\sum_{j} \delta\left(t-t_{j}\right)}{\left|\dot{x}\left(t_{j}\right)\right|}
$$

and by manipulation of equation (55), one obtains

$$
\lim _{l \rightarrow 0} \psi(t)=\pi \sum_{j} \Theta\left(t-t_{j}\right)
$$

where $\mathrm{d} \Theta / \mathrm{d} t=\delta(t)$ and $\Theta(t)$ is the Heaviside step function.

This last equation is tested numerically by integration of system of equation (37) for two values of $l$, being the results given in Figure (6). Upon decreasing value of $l$, excellent results are found for the phase $\psi$.

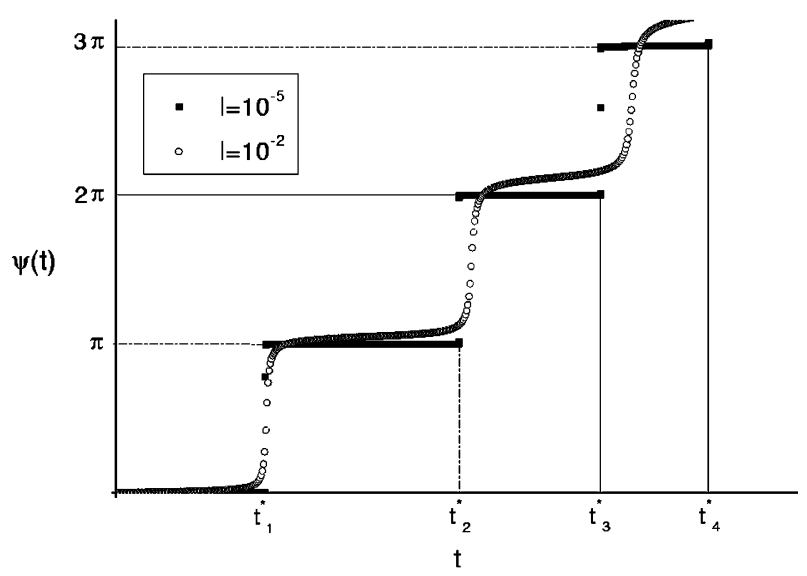

Figure 6. Numerical test for the phase $\psi$ in equation (58) for $\ell=10^{-5}$ and $\ell=10^{-2}$. 


\section{References}

1. Housner, W.G., 'The behavior of inverted pendulum structures during earthquakes', Bull. Seismol. Soc. Amer. 53 (1963) 403-417.

2. Aslam, M., Godden, W.G. and Scalise, D.T., 'Earthquake rocking response of rigid bodies', J. Struct. Div. 106(2) (1980) 377-392.

3. Spanos, P.D. and Koh, A.S., 'Rocking of rigid blocks due to harmonic shaking', J. Engrg. Mech. Div., ASCE, 110(11) (1984) 1672-1642.

4. Yim, C.S., Chopra, A.K. and Penzien, J., 'Rocking response of rigid blocks to earthquakes', Earth. Eng. and Struct. Dyn. 8 (1980) 565-587.

5. Makris, N. and Roussos, Y.S., 'Rocking response of rigid blocks under near-source ground motions', Geotéchnique 50 (1999) No. 3 243-262.

6. Hogan, S.J., 'On the dynamics of rigid block motion under harmonic forcing', Proc. R. Soc. Lond. A 425 (1989) 441-476.

7. Yim, S.C.S. and Lin, H., 'Nonlinear impact and chaotic response of slender rocking objects', $J$. Eng. Mech. 117 (1991) 2079-2100.

8. Augusti, G. and Sinopoli, A., 'Modelling the dynamics of large block structures', Meccanica 27 (1992) 195-211.

9. Psycharis, I.N., 'Dynamic behavior of rocking two-block assemblies', Earth. Eng. Struct. Dyn. 19 $1990555-575$.

10. Lipscombe, P.R., Dynamics of rigid block structures, Dissertation submitted to the University of Cambridge for the degree of Doctor of Philosophy (1990).

11. Lanczos, C. The Variational Principles of Mechanics. University of Toronto Press, Toronto, 1949.

12. Goldstein, H., Classical Mechanics. Addison-Wesley, Reading, Massachusetts, 1950.

13. Prieto, F., Lourenço, P.B. and Oliveira, C.S., 'Impulsive Dirac-delta forces in the Rocking Motion', Earth. Eng and Struct. Dyn. 33 (2004) 839-857.

14. Arfken, G.B. and Weber, H.J. Mathematical Methods for Physicists, 4th edn, Academic Press, 1995, pp. 81-87. 